\title{
The Hamiltonian Structure and Algebrogeometric Solution of a 1 + 1-Dimensional Coupled Equations
}

\author{
Hongfei Pan and Tiecheng Xia \\ Department of Mathematics, Shanghai University, Shanghai 200444, China \\ Correspondence should be addressed to Tiecheng Xia; xiatc@shu.edu.cn
}

Received 20 May 2013; Accepted 24 August 2013

Academic Editor: Jinde Cao

Copyright (C) 2013 H. Pan and T. Xia. This is an open access article distributed under the Creative Commons Attribution License, which permits unrestricted use, distribution, and reproduction in any medium, provided the original work is properly cited.

A 1+1-dimensional coupled soliton equations are decomposed into two systems of ordinary differential equations. The Abel-Jacobi coordinates are introduced to straighten the flows, from which the algebrogeometric solutions of the coupled $1+1$-dimensional equations are obtained in terms of the Riemann theta functions.

\section{Introduction}

The study of explicit solutions for soliton equations is very important in modern mathematics, physics, and other sciences. There are several systematic approaches to obtain explicit solutions of the soliton equations, such as the inverse scattering transformation, the algebrogeometric method, and the Hirota bilinear method (see, e.g., [1-4] and references therein). Algebrogeometric (or quasiperiodic) solutions are very important explicit solutions for soliton equations, recently, based on the nonlinearization technique of Lax pairs and direct method proposed by Cao [5]. This new scheme is further shown to be a very powerful tool, through which algebrogeometric solutions of $(1+1)$-dimensional and $(2+1)$ dimensional continuous and discrete soliton equations can be obtained; see Cao et al. [6, 7], Geng et al. [8, 9], and Dai and Fan [10].

In this paper, we will construct the Hamiltonian structure and search for the algebrogeometric solution of the following coupled 1+1-dimensional soliton equations:

$$
\begin{aligned}
& q_{t}=r_{x x}-3 q^{2} r_{x}+q_{x} r^{2}+2 q r r_{x}, \\
& r_{t}=q_{x x}+3 r^{2} r_{x}-r_{x} q^{2}-2 q r q_{x} .
\end{aligned}
$$

Our purpose is to construct the Hamiltonian structure and give the algebrogeometric solutions of the coupled $1+$ 1-dimensional soliton equations based on its obtained Lax pairs. The paper is organized as follows. In Section 2, we use Lenard operator pairs to derive another form of the coupled $1+1$-dimensional soliton equations. In Section 3, based on the trace identity $[11,12]$, we construct the Hamiltonian structure of the coupled 1+1-dimensional soliton equations. In Section 4, based on the Lax pairs of the coupled $1+1$ dimensional soliton equations, variable separation technique is used to translate the solution of the coupled $1+1$ dimensional soliton equations to solve ordinary differential equations. In Section 5, a hyperelliptic Riemann surface of genus $N$ and Abel-Jacobi coordinates are defined to straighten the associated flows. Jacobi's inverse problem is discussed, from which the algebrogeometric solutions of the coupled $1+1$-dimensional soliton equations are constructed in terms of the Riemann theta functions.

\section{The Hierarchy and Lax Pairs of the Coupled $1+1$-Dimensional Soliton Equations}

In this section, we introduce the Lenard gradient sequence $\left\{S_{j}\right\}_{j=0,1,2 \ldots}$ to derive the hierarchy and its stationary hierarchy associated with (1) by the recursion relation:

$$
\begin{gathered}
K S_{j-1}=J S_{j}, \quad j=1,2,3,\left.\ldots \quad S_{j}\right|_{(u, v)=0}=0, \\
S_{0}=(q-r, q+r, 1)^{T},
\end{gathered}
$$


where $S_{j}=\left(S_{j}^{(1)}, S_{j}^{(2)}, S_{j}^{(3)}\right)$ and operators $(\partial=\partial / \partial x)$

$$
J=\left(\begin{array}{ccc}
-2 & 0 & 2(q-r) \\
0 & 2 & -2(q+r) \\
-(q+r) & q-r & \partial
\end{array}\right)
$$

$$
K=\left(\begin{array}{ccc}
\partial & 0 & 0 \\
0 & \partial & 0 \\
-(q+r) & q-r & \partial
\end{array}\right)
$$

A direct calculation gives from the recursion relation (2) that

$$
\begin{gathered}
S_{1}=\left(\begin{array}{c}
-\frac{1}{2}(q+r)(q-r)^{2}-\frac{1}{2}\left(q_{x}-r_{x}\right) \\
-\frac{1}{2}(q+r)^{2}(q-r)+\frac{1}{2}\left(q_{x}+r_{x}\right) \\
-\frac{1}{2}(q+r)(q-r)
\end{array}\right), \\
S_{2}=\left(\begin{array}{c}
\frac{3}{8}(q+r)^{2}(q-r)^{3}+\frac{1}{2} r(q-r)\left(q_{x}-r_{x}\right)+\frac{1}{2}(q+r)(q-r)\left(q_{x}-r_{x}\right) \\
+\frac{1}{4}\left(q_{x x}-r_{x x}\right)+\frac{1}{4}\left(q_{x}+r_{x}\right)(q-r)^{2}-\frac{1}{2}(q-r) \partial^{-1}\left(q_{x}-r_{x}\right) r_{x} \\
\frac{3}{8}(q+r)^{3}(q-r)^{2}+\frac{1}{2} r(q+r)\left(q_{x}-r_{x}\right)-\frac{1}{2}(q+r)(q-r)\left(q_{x}+r_{x}\right) \\
-\frac{1}{4}\left(q_{x x}-r_{x x}\right)-\frac{1}{4}\left(q_{x}-r_{x}\right)(q+r)^{2}-\frac{1}{2}(q+r) \partial^{-1}\left(q_{x}-r_{x}\right) r_{x} \\
\frac{3}{8}(q+r)^{2}(q-r)^{2}+\frac{1}{2} r\left(q_{x}-r_{x}\right)-\frac{1}{2} \partial^{-1}\left(q_{x}-r_{x}\right) r_{x}
\end{array}\right) .
\end{gathered}
$$

Consider the spectral problem

$$
\psi_{x}=U \psi, \quad U=\left(\begin{array}{cc}
\lambda & q+r \\
\lambda(q-r) & -\lambda
\end{array}\right),
$$

and the auxiliary problem

$$
\psi_{t_{m}}=V^{(m)} \psi, \quad V^{(m)}=\left(\begin{array}{cc}
V_{11}^{(m)} & V_{12}^{(m)} \\
V_{21}^{(m)} & -V_{11}^{(m)}
\end{array}\right),
$$

where

$$
\begin{gathered}
V_{11}^{(m)}=\sum_{j=0}^{m} S_{j}^{(3)} \lambda^{m+1-j}, \\
V_{12}^{(m)}=\sum_{j=0}^{m} S_{j}^{(2)} \lambda^{m-j}, \\
V_{21}^{(m)}=\sum_{j=0}^{m} S_{j}^{(1)} \lambda^{m+1-j} .
\end{gathered}
$$

Then the compatibility condition of (5) and (6) is $U_{t_{m}}$ $V_{x}^{(m)}+\left[U, V^{(m)}\right]=0$, which is equivalent to the hierarchy of nonlinear evolution equations

$$
\begin{aligned}
& q_{t_{m}}=\frac{1}{2}\left(S_{m x}^{(2)}+S_{m x}^{(1)}\right), \\
& r_{t_{m}}=\frac{1}{2}\left(S_{m x}^{(2)}-S_{m x}^{(1)}\right) .
\end{aligned}
$$

In brief,

$$
\left(q_{t_{m}}, r_{t_{m}}\right)^{T}=X_{m}, \quad m \geq 0,
$$

$$
X_{m}=\left(\begin{array}{cc}
\partial & \partial \\
\partial & -\partial
\end{array}\right)\left(\begin{array}{c}
S_{m}^{(2)} \\
S_{m}^{(1)}
\end{array}\right) \text {. }
$$

The first two nontrivial equations are

$$
\begin{gathered}
q_{t_{0}}=q_{x}, \quad r_{t_{0}}=r_{x}, \\
q_{t_{1}}=r_{x x}-3 q^{2} r_{x}+q_{x} r^{2}+2 q r r_{x}, \\
r_{t_{1}}=q_{x x}+3 r^{2} r_{x}-r_{x} q^{2}-2 q r q_{x} .
\end{gathered}
$$

The second system is our coupled $1+1$-dimensional soliton equations (1).

Let $\psi=\left(\psi_{1}, \psi_{2}\right)^{T}$ and $\phi=\left(\phi_{1}, \phi_{2}\right)^{T}$ be two basic solutions of the spectral equations (5) and (6). We define a matrix $W$ by

$$
W=\frac{1}{2}\left(\phi \psi^{T}+\psi \phi^{T}\right) \sigma=\left(\begin{array}{cc}
f & g \\
h & -f
\end{array}\right), \quad \sigma=\left(\begin{array}{cc}
0 & -1 \\
1 & 0
\end{array}\right),
$$

in which $f, g$, and $h$ are three functions. It is easy to calculate by (5) and (6) that

$$
W_{x}=[U, W], \quad W_{t_{m}}=\left[V^{(m)}, W\right],
$$


which implies that $\partial_{x} \operatorname{det} W=0, \partial_{t_{m}} \operatorname{det} W=0$. Equation (13) can be written as

$$
\begin{gathered}
f_{x}=-\lambda(q-r) g+(q+r) h, \\
g_{x}=-2(q+r) f+2 \lambda g, \\
h_{x}=2 \lambda(q-r) f-2 \lambda h, \\
f_{t_{m}}=h V_{12}^{(m)}-g V_{21}^{(m)}, \\
g_{t_{m}}=2 g V_{11}^{(m)}-2 f V_{12}^{(m)}, \\
h_{t_{m}}=2 f V_{21}^{(m)}-2 h V_{11}^{(m)} .
\end{gathered}
$$

We suppose that the functions $f, g$, and $h$ are finite-order polynomials in $\lambda$ :

$$
f=\sum_{j=0}^{N} f_{j} \lambda^{N-j+1}, \quad g=\sum_{j=0}^{N} g_{j} \lambda^{N-j}, \quad h=\sum_{j=0}^{N} h_{j} \lambda^{N-j} .
$$

Substituting (16) into (14) yields

$$
\begin{gathered}
K G_{j-1}=J G_{j}(j=1,2, \ldots, N), \quad J G_{0}=0, \\
K G_{N}=0, \quad G_{j}=\left(h_{j}, g_{j}, f_{j}\right)^{T} .
\end{gathered}
$$

It is easy to see that (17) implies

$$
-(q+r) h_{j}+(q-r) g_{j}+f_{j x}=0
$$

and the equation $J G_{0}=0$ has the general solution

$$
G_{0}=\alpha_{0} S_{0},
$$

where $\alpha_{0}$ is constant of integration. Therefore, if we take (19) as a starting point, then $G_{j}$ can be determined recursively by relation (17). In fact, noticing ker $J=\left\{c S_{0} \mid \forall c \in \mathbb{R}\right\}$ and acting with the operator $\left(J^{-1} K\right)^{k}$ upon (19), we obtain from (2) and (17) that

$$
G_{k}=\sum_{j=0}^{k} \alpha_{j} S_{k-j}, \quad k=0,1, \ldots, N,
$$

where $\alpha_{0}, \alpha_{1}, \ldots, \alpha_{k}$ are integral constants. Substituting (20) into (17) yields a certain stationary evolution equation:

$$
\alpha_{0} \widehat{X}_{N}+\alpha_{1} \widehat{X}_{N-1}+\cdots+\alpha_{N} \widehat{X}_{0}=0
$$

where

$$
\widehat{X}_{j}=\left(\begin{array}{ll}
\partial & 0 \\
0 & \partial
\end{array}\right)\left(\begin{array}{c}
S_{j}^{(1)} \\
S_{j}^{(2)}
\end{array}\right) .
$$

This means that expression (16) is existent.

\section{Hamiltonian Structure}

Let

$$
V=V=\left(\begin{array}{cc}
V_{11} & V_{12} \\
V_{21} & -V_{11}
\end{array}\right)
$$

where

$$
\begin{gathered}
V_{11}=\sum_{j \geq 0} S_{j}^{(3)} \lambda^{-j+1}, \quad V_{12}=\sum_{j \geq 0} S_{j}^{(2)} \lambda^{-j}, \\
V_{21}=\sum_{j \geq 0} S_{j}^{(1)} \lambda^{-j+1} .
\end{gathered}
$$

It is easy to calculate

$$
\begin{aligned}
\operatorname{tr}\left(V \frac{\partial U}{\partial \lambda}\right) & =2 V_{11}+(q-r) V_{12} \\
& =\sum_{j \geq 1}\left(2 S_{j}^{(3)}+(q-r) S_{j-1}^{(2)}\right) \lambda^{-j+1}+2 S_{0}^{(3)} \lambda, \\
\operatorname{tr}\left(V \frac{\partial U}{\partial q}\right) & =\lambda V_{12}+V_{21}=\sum_{j \geq 0}\left(S_{j}^{(2)}+S_{j}^{(1)}\right) \lambda^{-j+1},
\end{aligned}
$$

$$
\operatorname{tr}\left(V \frac{\partial U}{\partial r}\right)=-\lambda V_{12}+V_{21}=\sum_{j \geq 0}\left(-S_{j}^{(2)}+S_{j}^{(1)}\right) \lambda^{-j+1} .
$$

According to the trace identity $[11,12]$, we have

$$
\left(\begin{array}{c}
\frac{\delta}{\delta q} \\
\frac{\delta}{\delta r}
\end{array}\right)\left(2 V_{11}+(q-r) V_{12}\right)=\left(\lambda^{-s} \frac{\partial}{\partial \lambda} \lambda^{s}\right)\left(\begin{array}{c}
\lambda V_{12}+V_{21} \\
-\lambda V_{12}+V_{21}
\end{array}\right)
$$

Comparing the coefficients of $\lambda^{-j+1}$, we obtain

$$
\left(\begin{array}{c}
\frac{\delta}{\delta q} \\
\frac{\delta}{\delta r}
\end{array}\right)\left(2 S_{j}^{(3)}+(q-r) S_{j-1}^{(2)}\right)=(-j+2+s)\left(\begin{array}{c}
S_{j-1}^{(2)}+S_{j-1}^{(1)} \\
-S_{j-1}^{(2)}+S_{j-1}^{(1)}
\end{array}\right),
$$

we set $j=1$ and then get $s=-1$ and

$$
\left(\begin{array}{c}
\frac{\delta}{\delta q} \\
\frac{\delta}{\delta r}
\end{array}\right) \mathscr{H}_{j}=\left(\begin{array}{c}
S_{j-1}^{(2)}+S_{j-1}^{(1)} \\
-S_{j-1}^{(2)}+S_{j-1}^{(1)}
\end{array}\right)=\left(\begin{array}{cc}
1 & 1 \\
-1 & 1
\end{array}\right)\left(\begin{array}{c}
S_{j-1}^{(2)} \\
S_{j-1}^{(1)}
\end{array}\right),
$$

where $\mathscr{H}_{j}=\left(2 S_{j}^{(3)}+(q-r) S_{j-1}^{(2)}\right) /(-j+1)$.

Thus the soliton equation (9) has a Hamiltonian structure:

$$
\left(\begin{array}{l}
q \\
r
\end{array}\right)_{t_{m}}=\frac{1}{2}\left(\begin{array}{cc}
\partial & \partial \\
\partial & -\partial
\end{array}\right)\left(\begin{array}{c}
S_{m}^{(2)} \\
S_{m}^{(1)}
\end{array}\right)=\widetilde{J}\left(\begin{array}{c}
\frac{\delta}{\delta q} \\
\frac{\delta}{\delta r}
\end{array}\right) \mathscr{H}_{m+1}, \quad m \geq 0,
$$


where

$$
\widetilde{J}=\frac{1}{2}\left(\begin{array}{cc}
\partial & 0 \\
0 & -\partial
\end{array}\right) .
$$

In speciality, the Hamiltonian structure of $(1)$ is $(m=1)$ :

$$
\begin{aligned}
\mathscr{H}_{2}= & \frac{1}{8}(q+r)^{2}(q-r)^{2}-\frac{q q_{x}-2 r r_{x}+q r_{x}}{2} \\
& +\frac{\partial^{-1}\left(q_{x}-r_{x}\right) r_{x}}{2} .
\end{aligned}
$$

\section{Ordinary Differential Equations}

In this section, (1) will be decomposed into two systems of solvable ordinary differential equations. Without loss of generality, let $\alpha_{0}=1$. From (2), (17), and (20), we have

$$
\begin{gathered}
f_{0}=1, \quad g_{0}=q+r, \quad h_{0}=q-r, \\
f_{1}=-\frac{1}{2}(q+r)(q-r)+\alpha_{1}, \\
g_{1}=-\frac{1}{2}(q+r)^{2}(q-r)+\frac{1}{2}\left(q_{x}+r_{x}\right)+\alpha_{1}(q+r), \\
h_{1}=-\frac{1}{2}(q+r)(q-r)^{2}-\frac{1}{2}\left(q_{x}-r_{x}\right)+\alpha_{1}(q-r) .
\end{gathered}
$$

By using (16), we can write $g$ and $h$ as the following finite products:

$$
\begin{gathered}
g=-(q+r) \prod_{j=1}^{N}\left(\lambda-u_{j}\right), \\
h=(q-r) \prod_{j=1}^{N}\left(\lambda-v_{j}\right) .
\end{gathered}
$$
$\lambda^{N-1}$ that

Equation (33) implies by comparing the coefficients of

$$
g_{1}=-(q+r) \sum_{j=1}^{N} u_{j}, \quad h_{1}=-(q+r) \sum_{j=1}^{N} v_{j} .
$$

Thus from (32) and (34), we obtain

$$
\begin{aligned}
& -\frac{1}{2}(q+r)^{2}(q-r)+\frac{1}{2}\left(q_{x}+r_{x}\right)+\alpha_{1}(q+r) \\
& =-(q+r) \sum_{j=1}^{N} u_{j}, \\
& -\frac{1}{2}(q+r)(q-r)^{2}-\frac{1}{2}\left(q_{x}-r_{x}\right)+\alpha_{1}(q-r) \\
& =-(q-r) \sum_{j=1}^{N} v_{j} .
\end{aligned}
$$

Let us consider the function $\operatorname{det} W$ which is a $(2 N+2)$ thorder polynomial in $\lambda$ with constant coefficients of the $x$-flow and $t_{m}$-flow:

$$
-\operatorname{det} W=f^{2}+g h=\prod_{j=1}^{2 N+2}\left(\lambda-\lambda_{j}\right)=R(\lambda) .
$$

Substituting (16) into (36) and comparing the coefficients of $\lambda^{2 N+1}$ yield

$$
2 f_{0} f_{1}+g_{0} h_{0}=-\sum_{j=1}^{2 N+2} \lambda_{j}
$$

which together with (32) gives

$$
\alpha_{1}=-\frac{1}{2} \sum_{j=1}^{2 N+2} \lambda_{j}
$$

From (36) we see that

$$
\left.f\right|_{\lambda=u_{k}}=\sqrt{R\left(u_{k}\right)},\left.\quad f\right|_{\lambda=v_{k}}=\sqrt{R\left(v_{k}\right)} .
$$

Again by using (14) and (33), we obtain

$$
\begin{aligned}
& \left.g_{x}\right|_{\lambda=u_{k}}=-(q+r) u_{k x} \prod_{j=1, j \neq k}^{N}\left(u_{k}-u_{j}\right)=-\left.2(q+r) f\right|_{\lambda=u_{k}}, \\
& \left.h_{x}\right|_{\lambda=v_{k}}=-(q-r) v_{k x} \prod_{j=1, j \neq k}^{N}\left(v_{k}-v_{j}\right)=\left.2 v_{k}(q-r) f\right|_{\lambda=v_{k}},
\end{aligned}
$$

which together with (39) gives

$$
\begin{array}{ll}
u_{k x}=\frac{2 \sqrt{R\left(u_{k}\right)}}{\prod_{j=1, j \neq k}^{N}\left(u_{k}-u_{j}\right)}, \quad 1 \leq k \leq N, \\
v_{k, x}=\frac{-2 \sqrt{R\left(v_{k}\right)}}{\prod_{j=1, j \neq k}^{N}\left(v_{k}-v_{j}\right)}, \quad 1 \leq k \leq N .
\end{array}
$$


In a way similar to the above expression, by using (6) $(m=$ $\left.1, t_{1}=t\right)$, (15), and (39), we arrive at the evolution of $\left\{u_{k}\right\}$ and $\left\{v_{k}\right\}$ along the $t_{m}$-flow:

$$
\begin{aligned}
u_{k, t} & =\frac{\left.\left.2 f\right|_{\lambda=u_{k}} V_{12}^{(1)}\right|_{\lambda=u_{k}}}{(q+r) \prod_{j=1, j \neq k}^{N}\left(u_{k}-u_{j}\right)} \\
& =\frac{2 \sqrt{R\left(u_{k}\right)}\left[u_{k}-(1 / 2)(q+r)(q-r)+(1 / 2) \partial \ln (q-r)\right]}{\prod_{j=1, j \neq k}^{N}\left(u_{k}-u_{j}\right)},
\end{aligned}
$$

$$
\begin{aligned}
& v_{k, t} \\
& =-\frac{\left.\left.2 f\right|_{\lambda=v_{k}} V_{21}^{(1)}\right|_{\lambda=v_{k}}}{(q-r) \prod_{j=1, j \neq k}^{N}\left(v_{k}-v_{j}\right)} \\
& =\left(-2 \sqrt{R\left(v_{k}\right)}\left[v_{k}^{2}-\frac{1}{2}(q+r)(q-r) v_{k}\right.\right. \\
& \left.\left.\quad+\frac{1}{2} \partial \ln (q-r) v_{k}\right]\right)\left(\prod_{j=1, j \neq k}^{N}\left(v_{k}-v_{j}\right)\right)^{-1} .
\end{aligned}
$$

Therefore, if the $(2 N+2)$ distinct parameters $\lambda_{1}, \lambda_{2}, \ldots, \lambda_{2 N+2}$ are given and let $u_{k}(x, t)$ and $v_{k}(x, t)$ be distinct solutions of ordinary differential equations (41), (42), and (43), then $(q, r)$ determined by (35) is a solution of the coupled $1+1$-dimensional equations (1).

\section{Algebrogeometric Solutions}

In this section, we will give the algebrogeometric solutions of the coupled $1+1$-dimensional equation (1). To this end, we first introduce the Riemann surface $\Gamma$ of the hyperelliptic curve

$$
\Gamma: \zeta^{2}=R(\lambda), \quad R(\lambda)=\prod_{j=1}^{2 N+2}\left(\lambda-\lambda_{j}\right),
$$

with genus $N$ on $\Gamma$. On $\Gamma$ there are two infinite points $\infty_{1}$ and $\infty_{2}$, which are not branch points of $\Gamma$. We equip $\Gamma$ with a canonical basis of cycles: $a_{1}, a_{2}, \ldots, a_{N} ; b_{1}, b_{2}, \ldots, b_{N}$ which are independent and have intersection numbers as follows:

$$
\begin{gathered}
a_{i} \circ a_{j}=0, \quad b_{i} \circ b_{j}=0, \\
a_{i} \circ b_{j}=\delta_{i j}, \quad i, j=1,2, \ldots, N .
\end{gathered}
$$

We will choose the following set as our basis:

$$
\widetilde{\omega}_{l}=\frac{\lambda^{l-1} d \lambda}{\sqrt{R(\lambda)}}, \quad l=1,2, \ldots, N
$$

which are linearly independent of each other on $\Gamma$, and let

$$
A_{i j}=\int_{a_{j}} \widetilde{\omega}_{i}, \quad B_{i j}=\int_{b_{j}} \widetilde{\omega}_{i} .
$$

It is possible to show that the matrices $A=\left(A_{i j}\right)$ and $B=$ $\left(B_{i j}\right)$ are $N \times N$ invertible matrices $[13,14]$. Now we define the matrices $C$ and $\tau$ by $C=\left(C_{i j}\right)=A^{-1}, \tau=\left(\tau_{i j}\right)=A^{-1} B$. Then the matrix $\tau$ can be shown to be symmetric $\left(\tau_{i j}=\tau_{j i}\right)$ and it has a positive-definite imaginary part ( $\operatorname{Im} \tau>0)$. If we normalize $\widetilde{\omega}_{j}$ into the new basis $\omega_{j}$

$$
\omega_{j}=\sum_{l=1}^{N} C_{j l} \widetilde{\omega}_{l}, \quad l=1,2, \ldots, N
$$

then we have

$$
\begin{gathered}
\int_{a_{j}} \omega_{j}=\sum_{l=1}^{N} C_{j l} \int_{a_{j}} \widetilde{\omega}_{l}=\sum_{l=1}^{N} C_{j l} A_{l i}=\delta_{j i}, \\
\int_{b_{j}} \omega_{i}=\sum_{l=1}^{N} C_{j l} \int_{b_{j}} \bar{\omega}_{l}=\sum_{l=1}^{N} C_{j l} B_{l i}=\tau_{j i} .
\end{gathered}
$$

Now we introduce the Abel-Jacobi coordinates as follows:

$$
\begin{aligned}
& \rho_{j}^{(1)}(x, t)=\sum_{k=1}^{N} \int_{p_{0}}^{p\left(u_{k}(x, t)\right)} \omega_{j}=\sum_{k=1}^{N} \sum_{l=1}^{N} \int_{\lambda\left(p_{0}\right)}^{u_{k}} C_{j l} \frac{\lambda^{l-1} d \lambda}{\sqrt{R(\lambda)}} \\
& \rho_{j}^{(2)}(x, t)=\sum_{k=1}^{N} \int_{p_{0}}^{p\left(v_{k}(x, t)\right)} \omega_{j}=\sum_{k=1}^{N} \sum_{l=1}^{N} \int_{\lambda\left(p_{0}\right)}^{v_{k}(x, t)} C_{j l} \frac{\lambda^{l-1} d \lambda}{\sqrt{R(\lambda)}},
\end{aligned}
$$

where $p\left(u_{k}(x, t)\right)=\left(u_{k}, \sqrt{R\left(u_{k}\right)}\right), p\left(v_{k}(x, t)\right)=\left(v_{k}, \sqrt{R\left(v_{k}\right)}\right)$, and $\lambda\left(p_{0}\right)$ is the local coordinate of $p_{0}$. From (42) and (50), we get

$$
\partial_{x} \rho_{j}^{(1)}=\sum_{k=1}^{N} \sum_{l=1}^{N} C_{j l} \frac{u_{k}^{l-1} u_{k x}}{\sqrt{R\left(u_{k}\right)}}=\sum_{k=1}^{N} \sum_{l=1}^{N} \frac{C_{j l} u_{k}^{l-1}}{\prod_{j=1, j \neq k}^{N}\left(u_{k}-u_{j}\right)},
$$

which implies

$$
\partial_{x} \rho_{j}^{(1)}=2 C_{j N}=\Omega_{j}^{(1)}, \quad j=1,2, \ldots, N .
$$

With the help of the following equality

$$
\sum_{k=1}^{N} \frac{u_{k}^{l-1}}{\prod_{j=1, j \neq k}^{N}\left(u_{k}-u_{j}\right)}=\delta_{l N}, \quad l=1,2, \ldots, N
$$

in a similar way, we obtain from (50), (51), (41), (42), and (43) that

$$
\begin{gathered}
\partial_{t} \rho_{j}^{(1)}=2 \sum_{l=1}^{N} C_{j l}\left(\sum_{i=1}^{N} u_{i}\right)-C_{j N}(q+r)(q-r) \\
+C_{j N} \partial \ln (q-r)=\Omega_{j}^{(2)}, \\
\partial_{x} \rho_{j}^{(2)}=-\Omega_{j}^{(1)}, \quad j=1,2, \ldots, N, \\
\partial_{t} \rho_{j}^{(2)}=-\Omega_{j}^{(2)}, \quad j=1,2, \ldots, N .
\end{gathered}
$$


On the basis of these results, we obtain the following:

$$
\begin{gathered}
\rho_{j}^{(1)}(x, t)=\Omega_{j}^{(1)} x+\Omega_{j}^{(2)} t+\gamma_{j}^{(1)}, \\
\rho_{j}^{(2)}(x, t)=-\Omega_{j}^{(1)} x-\Omega_{j}^{(2)} t+\gamma_{j}^{(2)},
\end{gathered}
$$

where $\gamma_{j}^{(i)}(i=1,2)$ are constants and

$$
\begin{gathered}
\gamma_{j}^{(1)}=\sum_{k=1}^{N} \int_{p_{0}}^{p\left(\widetilde{u}_{k}(0,0)\right)} \omega_{j}, \quad \gamma_{j}^{(2)}=\sum_{k=1}^{N} \int_{p_{0}}^{p\left(\widetilde{v}_{k}(0,0)\right)} \omega_{j}, \\
\rho^{(1)}=\left(\rho_{1}^{(1)}, \rho_{2}^{(1)}, \ldots, \rho_{N}^{(1)}\right)^{T}, \\
\rho^{(2)}=\left(\rho_{1}^{(2)}, \rho_{2}^{(2)}, \ldots, \rho_{N}^{(2)}\right)^{T}, \\
\Omega^{(m)}=\left(\Omega_{1}^{(m)}, \Omega_{2}^{(m)}, \ldots, \Omega_{N}^{(m)}\right)^{T}, \\
\gamma^{(m)}=\left(\gamma_{1}^{(m)}, \gamma_{2}^{(m)}, \ldots, \gamma_{N}^{(m)}\right)^{T}, \quad m=1,2 .
\end{gathered}
$$

Now we introduce the Abel map $\mathscr{A}(p)$ :

$$
\begin{gathered}
\mathscr{A}(p)=\int_{p_{0}}^{p} \omega, \quad \omega=\left(\omega_{1}, \omega_{2}, \ldots, \omega_{N}\right)^{T}, \\
\mathscr{A}\left(\sum_{k} p_{k}\right)=\sum n_{k} \mathscr{A}\left(p_{k}\right)
\end{gathered}
$$

and Abel-Jacobi coordinates:

$$
\begin{aligned}
& \rho^{(1)}=\mathscr{A}\left(\sum_{k=1}^{N} p\left(u_{k}\right)\right)=\sum_{k=1}^{N} \int_{p_{0}}^{p\left(u_{k}\right)} \omega, \\
& \rho^{(2)}=\mathscr{A}\left(\sum_{k=1}^{N} p\left(v_{k}\right)\right)=\sum_{k=1}^{N} \int_{p_{0}}^{p\left(v_{k}\right)} \omega .
\end{aligned}
$$

According to the Riemann theorem $[13,14]$, there exists a Riemann constant vector $M \in \mathbb{C}^{N}$ such that the function

$$
F^{(m)}(\lambda)=\theta\left(\mathscr{A}(p(\lambda))-\rho^{(m)}-M^{(m)}\right), \quad m=1,2,
$$

has exactly $N$ zeros at $u_{1}, u_{2}, \ldots, u_{N}$ for $m=1$ or $v_{1}, v_{2}, \ldots, v_{N}$ for $m=2$. To make the function single valued, the surface $\Gamma$ is cut along all $a_{k}, b_{k}$ to form a simple connected region, whose boundary is denoted by $\gamma$. By $[13,14]$, the integrals

$$
I(\Gamma)=\frac{1}{2 \pi i} \int_{\gamma} \lambda d \ln F^{(m)}, \quad m=1,2,
$$

are constants independent of $\rho^{(1)}$ and $\rho^{(2)}$ with

$$
I=I(\Gamma)=\sum_{j=1}^{N} \int_{a_{j}} \lambda \omega_{j}
$$

By the residue theorem, we have

$$
\begin{aligned}
& \sum_{j=1}^{N} u_{j}=I-\sum_{s=1}^{2} \operatorname{Res}_{\lambda=\infty_{s}} \lambda d \ln F^{(1)}(\lambda), \\
& \sum_{j=1}^{N} v_{j}=I-\sum_{s=1}^{2} \operatorname{Res}_{\lambda=\infty_{s}} \lambda d \ln F^{(2)}(\lambda) .
\end{aligned}
$$

Here we need only to compute the residues in (63). In a way similar to calculations in [10], we arrive at

$$
\begin{array}{r}
\operatorname{Res}_{\lambda=\infty_{s}} \lambda d \ln F^{(m)}(\lambda)=(-1)^{s+m} \partial \ln \theta_{s}^{(m)}, \\
m=1,2 ; s=1,2,
\end{array}
$$

where

$$
\begin{gathered}
\theta_{s}^{(1)}=\theta\left(\Omega^{(1)} x+\Omega^{(2)} t+\xi_{s}\right), \\
\theta_{s}^{(2)}=\theta\left(-\Omega^{(1)} x-\Omega^{(2)} t+\eta_{s}\right),
\end{gathered}
$$

and $\xi_{s}$ and $\eta_{s}$ are constants. Thus from (63) and (64), we arrive at

$$
\sum_{j=1}^{N} u_{j}=I-\partial \ln \frac{\theta_{1}^{(1)}}{\theta_{2}^{(1)}}, \quad \sum_{j=1}^{N} v_{j}=I-\partial \ln \frac{\theta_{2}^{(2)}}{\theta_{1}^{(2)}}
$$

Substituting (66) into (35), we get an algebrogeometric solution for the coupled $1+1$-dimensional soliton equations (1):

$$
\begin{array}{r}
q=\frac{A(t)}{2} \exp \left(-\partial^{-1} \sum_{j=1}^{2 N+2} \lambda_{j}+2 \partial^{-1} I-2 \ln \frac{\theta_{2}^{(2)}}{\theta_{1}^{(2)}}\right. \\
\left.-\partial^{-1} \exp \left(-2 \ln \frac{\theta_{2}^{(2)} \theta_{2}^{(1)}}{\theta_{1}^{(2)} \theta_{1}^{(1)}}\right)\right) \\
+\frac{A(t)}{2} \exp \left(\partial^{-1} \sum_{j=1}^{2 N+2} \lambda_{j}+2 \partial^{-1} I-2 \ln \frac{\theta_{1}^{(2)}}{\theta_{2}^{(1)}}\right. \\
\left.+\partial^{-1} \exp \left(-2 \ln \frac{\theta_{2}^{(2)} \theta_{2}^{(1)}}{\theta_{1}^{(2)} \theta_{1}^{(1)}}\right)\right),
\end{array}
$$

$$
\begin{array}{r}
r=\frac{A(t)}{2} \exp \left(\partial^{-1} \sum_{j=1}^{2 N+2} \lambda_{j}+2 \partial^{-1} I-2 \ln \frac{\theta_{1}^{(2)}}{\theta_{2}^{(1)}}\right. \\
\left.+\partial^{-1} \exp \left(-2 \ln \frac{\theta_{2}^{(2)} \theta_{2}^{(1)}}{\theta_{1}^{(2)} \theta_{1}^{(1)}}\right)\right) \\
-\frac{A(t)}{2} \exp \left(-\partial^{-1} \sum_{j=1}^{2 N+2} \lambda_{j}+2 \partial^{-1} I-2 \ln \frac{\theta_{2}^{(2)}}{\theta_{1}^{(2)}}\right. \\
\left.-\partial^{-1} \exp \left(-2 \ln \frac{\theta_{2}^{(2)} \theta_{2}^{(1)}}{\theta_{1}^{(2)} \theta_{1}^{(1)}}\right)\right),
\end{array}
$$

where $A(t)$ is arbitrary complex functions about variable $t$.

\section{Acknowledgments}

This work is in part supported by the Natural Science Foundation of China (Grant nos. 11271008, 61072147, and 11071159) and the Shanghai University Leading Academic Discipline Project (A.13-0101-12-004). 


\section{References}

[1] A. C. Newell, Solitons in Mathematics and Physics, vol. 48 of CBMS-NSF Regional Conference Series in Applied Mathematics, SIAM, Philadelphia, Pa, USA, 1985.

[2] M. J. Ablowitz and P. A. Clarkson, Solitons, Nonlinear Evolution Equations and Inverse Scattering, vol. 149 of London Mathematical Society Lecture Note Series, Cambridge University Press, 1991.

[3] B. A. Dubrovin, "Inverse problem for periodic finite-zoned potentials in the theory of scattering," Functional Analysis and Its Applications, vol. 9, no. 1, pp. 61-62, 1975.

[4] E. D. Belokolos, A. I. Bobenko, V. Z. Enolskii, A. R. Its, and V. B. Matveev, Algebro-Geometric Approach to Nonlinear Integrable Equations, Springer, Berlin, Germany, 1994.

[5] C. W. Cao, "Nonlinearization of the Lax system for AKNS hierarchy," Science in China A, vol. 33, no. 5, pp. 528-536, 1990.

[6] C. Cao, Y. Wu, and X. Geng, "Relation between the KadomtsevPetviashvili equation and the confocal involutive system," Journal of Mathematical Physics, vol. 40, no. 8, pp. 3948-3970, 1999.

[7] C. Cao, X. Geng, and H. Wang, "Algebro-geometric solution of the $2+1$ dimensional Burgers equation with a discrete variable," Journal of Mathematical Physics, vol. 43, no. 1, pp. 621-643, 2002.

[8] X. Geng, H. H. Dai, and C. Cao, "Algebro-geometric constructions of the discrete Ablowitz-Ladik flows and applications," Journal of Mathematical Physics, vol. 44, no. 10, pp. 4573-4588, 2003.

[9] X. Geng and C. Cao, "Explicit solutions of the 2+1-dimensional breaking soliton equation," Chaos, Solitons \& Fractals, vol. 22, no. 3, pp. 683-691, 2004.

[10] H. H. Dai and E. G. Fan, "Variable separation and algebro-geometric solutions of the Gerdjikov-Ivanov equation," Chaos, Solitons \& Fractals, vol. 22, no. 1, pp. 93-101, 2004.

[11] G. Z. Tu, "Infinitesimal canonical transformations of generalised Hamiltonian equations," Journal of Physics A, vol. 15, no. 1, pp. 277-285, 1982.

[12] G. Z. Tu, "The trace identity, a powerful tool for constructing the Hamiltonian structure of integrable systems," Journal of Mathematical Physics, vol. 30, no. 2, pp. 330-338, 1989.

[13] C. L. Siegel, Topics in Complex Function Theory, vol. 2, John Wiley \& Sons, New York, NY, USA, 1971.

[14] P. Griffiths and J. Harris, Principles of Algebraic Geometry, Wiley Classics Library, John Wiley \& Sons, New York, NY, USA, 1994. 


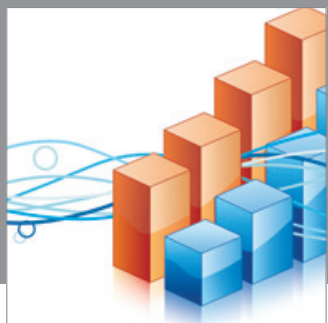

Advances in

Operations Research

mansans

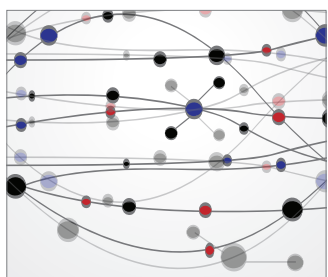

The Scientific World Journal
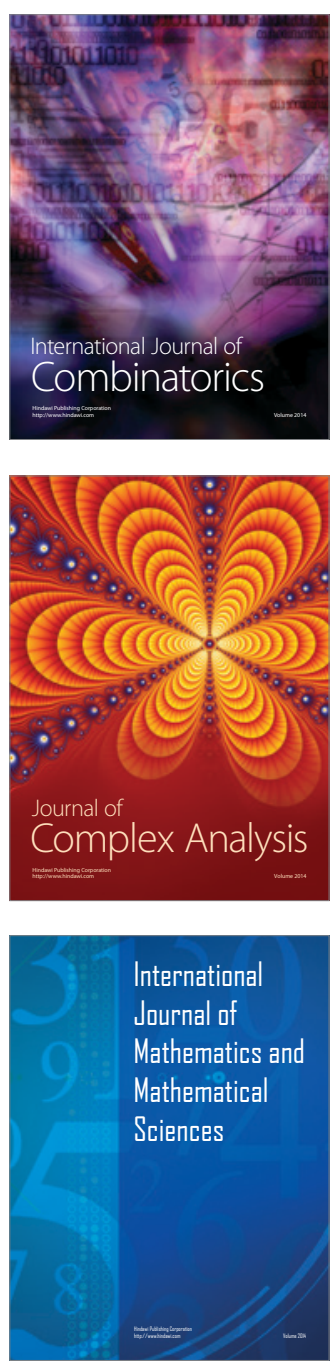
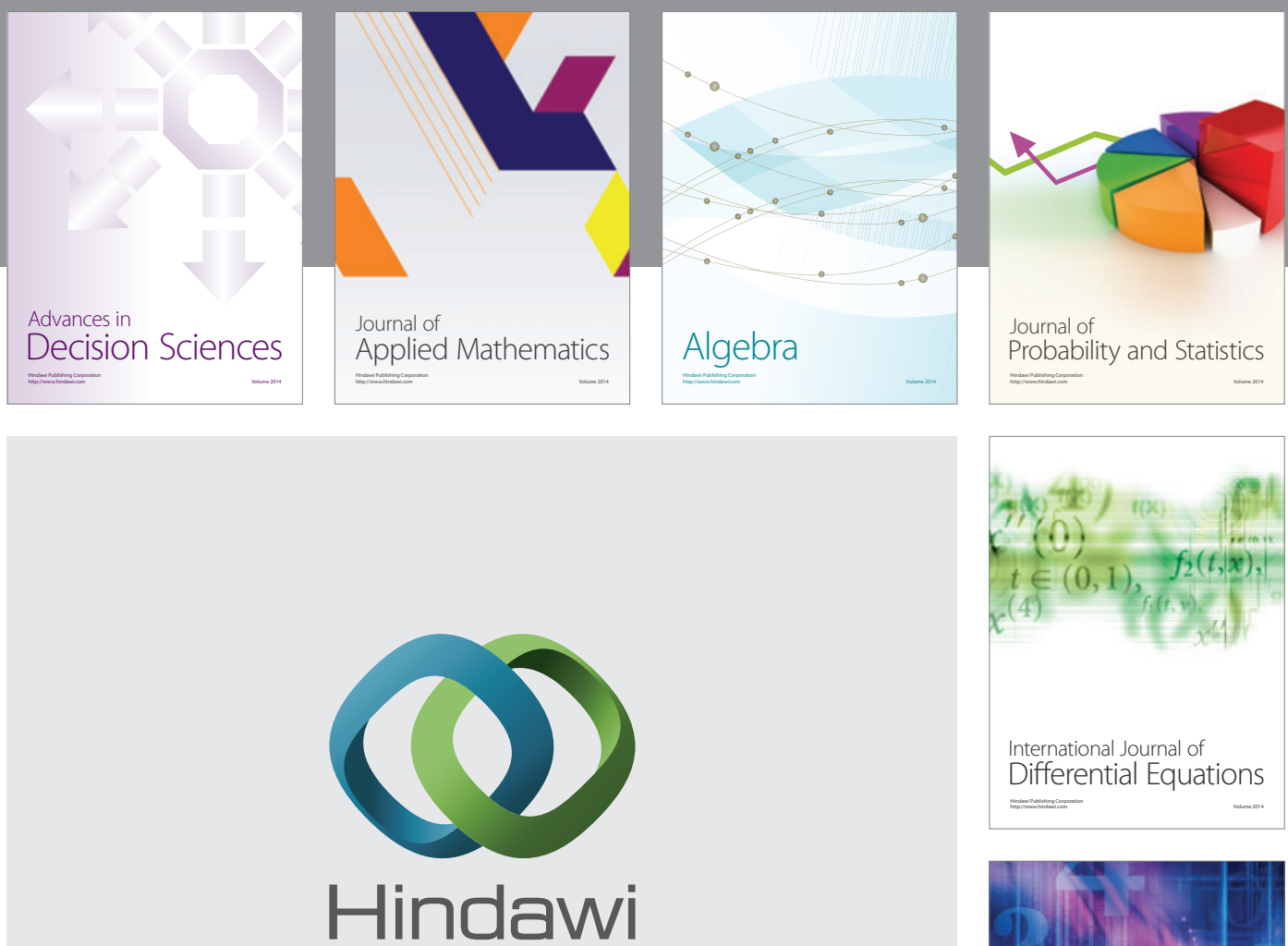

Submit your manuscripts at http://www.hindawi.com
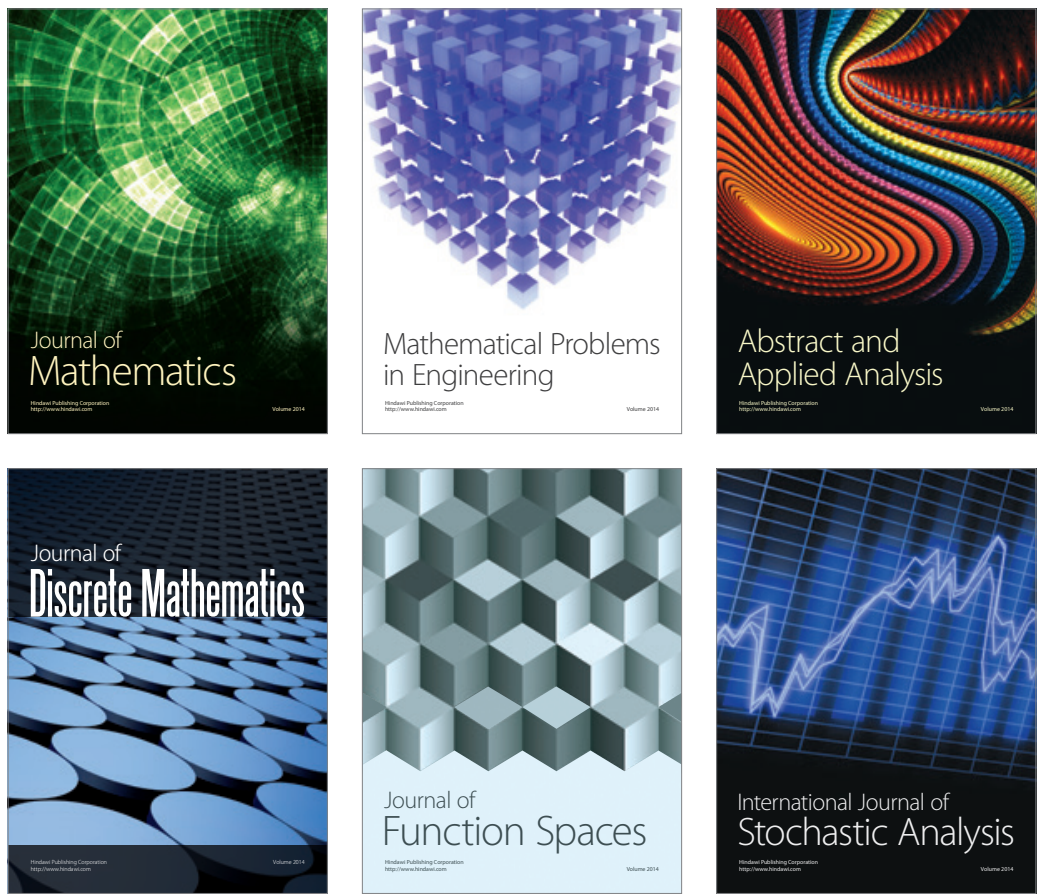

Journal of

Function Spaces

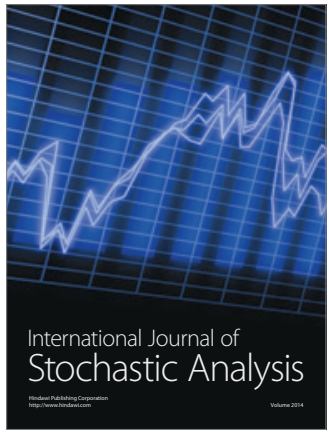

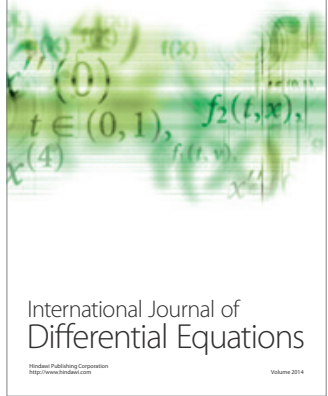
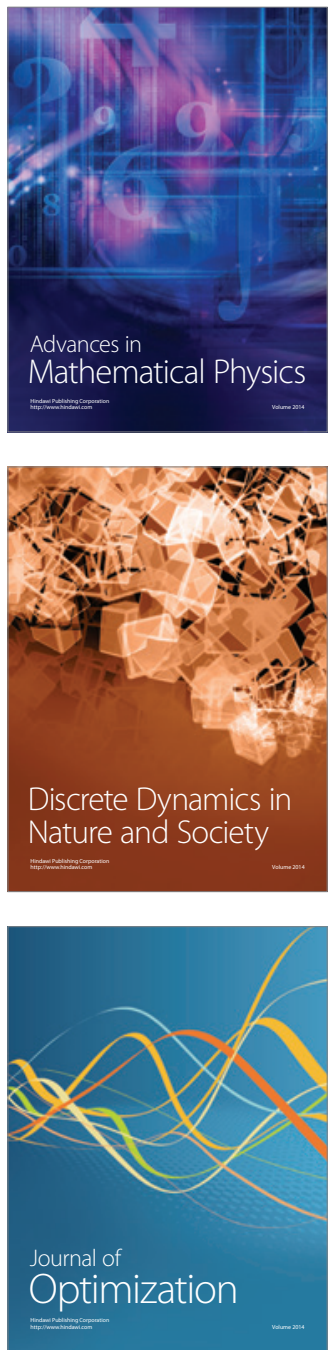